\title{
Involvement of Cytochrome P450 Metabolites in the Vascular Action of Angiotensin II on the Afferent Arterioles
}

\author{
Kentaro KOHAGURA, Shuji ARIMA, Yoshimi ENDO, Yoshiroh CHIBA, Osamu ITO, \\ Michiaki ABE, Ken OMATA, and Sadayoshi ITO
}

\begin{abstract}
Recent studies have demonstrated that cytochrome P450-dependent metabolites of arachidonic acid (CYP450-AA) play important roles in the control of renal vascular resistance (RVR). In the present study, we examined the possible involvement of CYP450-AA in the vasoconstrictor action of angiotensin II (Ang II) on the afferent arterioles (Af-Arts), a vascular segment crucial to the control of RVR. Rabbit Af-Arts were microperfused at $60 \mathrm{mmHg}$ in vitro, and the vasoconstrictor action of Ang II $\left(10^{-11-10^{-8}} \mathrm{M}\right.$, added to both the bath and lumen) was examined with or without blocking the activity of CYP450 epoxygenase or hydroxylase. Ang II decreased the luminal diameter of Af-Arts in a dose-dependent manner (34 $\pm 2 \%$ of control diameter at $10^{-8} \mathrm{M}, n=9, p<0.0001$ ). Pretreatment with miconazole, an inhibitor of CYP450 epoxygenase, at $10^{-6} \mathrm{M}$ decreased the basal diameter by $14 \pm 1 \%(n=6, p<0.01)$ and augmented the vasoconstrictor action of Ang II ( $7 \pm 3 \%$ of control diameter at $10^{-8} \mathrm{M}, p<0.001$ vs. without miconazole). This augmentation was abolished by blocking the Ang II type 2 (AT2) receptor with PD 123319 at $10^{-7} \mathrm{M}$. In contrast, pretreatment with 17-octadecynoic acid (17-ODYA, $10^{-8} \mathrm{M}$ ), which inhibits both epoxygenase and hydroxylase activity, had no effect on the basal diameter but attenuated the vasoconstrictor action of Ang II ( $46 \pm 2 \%$ of control diameter at $10^{-8} \mathrm{M}$, $p<0.01$ vs. without 17-ODYA). Our results demonstrate that in the Af-Art, endogenous CYP450-AA are involved not only in the control of basal tone but also in the action of Ang II. Further, it appears that the CYP450 epoxygenase pathway attenuates Ang II action via AT2 receptors. (Hypertens Res 2001; 24: 551-557)
\end{abstract}

Key Words: EET, 20-HETE, $\mathrm{AT}_{2}$ receptor, glomerular hemodynamics, microperfusion

\section{Introduction}

It is well known that angiotensin II (Ang II), the physiologically active component of the renin-angiotensin system, plays an important role in the regulation of renal vascular resistance (RVR) under both the physiological and pathological conditions (1). Abnormal RVR induced by altered renal vascular responses to Ang II is thought to be responsible, at least in part, for the development of several diseases, including hypertension (2). This idea has prompted many investi- gators, including us, to study factors that may affect the renal vascular responses to Ang II, and it has been demonstrated that the renal vasoconstrictor action of Ang II is modulated by endogenous vasodilators such as nitric oxide (NO) (3-5), prostaglandins $(3,4)$ and epoxyeicosatrienoic acids (EETs), which are cytochrome P450 (CYP450) epoxygenase-dependent arachidonates $(3,6)$. On the other hand, factors that potentiate the vasoconstrictor action of Ang II in the kidney has not been well defined.

Recent studies have suggested the possible existence of interactions between Ang II and CYP450-dependent metabo-

From the Division of Nephrology, Endocrinology and Vascular Medicine, Tohoku University School of Medicine, Sendai, Japan.

This work was supported in part by a research grant for cardiovascular research (11C-4) from the Ministry of Health and Welfare of Japan, and by grants (No.12470208 and 12877162) from the Ministry of Education, Science, and Culture, Japan. Results from this study were presented in part at the Council for High Blood Pressure Research 53rd Annual Fall Conference and Scientific Session (1999, Orlando).

Address for Reprints: Shuji Arima, M.D., Ph.D., Division of Nephrology, Endocrinology and Vascular Medicine, Tohoku University School of Medicine, 1-1 Seiryo-cho, Aoba-ku, Sendai 980-8574, Japan. E-mail: shuarima@mail.cc.tohoku.ac.jp

Received March 30, 2001; Accepted in revised form May 22, 2001. 
lites of arachidonic acid (CYP450-AA) in the kidney. Croft et al. (7) have demonstrated that Ang II increases the synthesis of 20-hydroxyeicosatetraenoic acid (20-HETE), a CYP450 hydroxylase-dependent arachidonate, in renal microvessels. 20-HETE is known to be a potent vasoconstrictor ( 6 ) and to increase the resistance of afferent arterioles (Af-Arts) through the tubuloglomerular feedback mechanism (TGF) (8) and through its direct vasoconstrictor action (9). Thus, it may be possible that in the Af-Art, Ang II stimulates the synthesis of 20-HETE, which in turn potentiates the vasoconstrictor action of Ang II on this vascular segment. In the present study, we directly examined the possible contribution of endogenous CYP450-AA, namely epoxygenase and hydroxylase metabolites, to the vasoconstrictor action of Ang II on the Af-Art. For this, we microdissected and perfused rabbit Af-Arts at constant pressure in vitro and studied whether inhibition of CYP450-AA synthesis affects the vasoconstrictor action of Ang II on the Af-Art.

\section{Methods}

\section{Isolation and Microperfusion of Rabbit Af-Arts}

This study was performed in accordance with the Guide for Animal Experimentation of the Tohoku University School of Medicine. We used previously described methods to isolate and microperfuse Af-Arts (10-12). Briefly, young male New Zealand white rabbits $(1.5-2.0 \mathrm{~kg})$, fed standard rabbit chow and tap water ad libitum, were anesthetized with intravenous sodium pentobarbital $(40 \mathrm{mg} / \mathrm{kg})$ and their kidneys were removed. From each rabbit, a single superficial Af-Art with its glomerulus (but not macula densa or other tubular segments) intact was microdissected under a stereomicroscope (SZH10; Olympus, Tokyo). Using a micropipette, the Af-Art was transferred to a temperature-regulated chamber mounted on an inverted microscope (IMT-2; Olympus). The Af-Art was then cannulated with an array of glass pipettes and perfused at $60 \mathrm{mmHg}$ throughout the experiments with oxygenated medium 199 (Gibco, Grand Island, USA) containing 5\% BSA (Sigma, St Louis, USA). The bath was identical to the arteriolar perfusate except that it contained $0.1 \%$ BSA, and was exchanged continuously. Microdissection and cannulation of the Af-Art were completed within $90 \mathrm{~min}$ at $8^{\circ} \mathrm{C}$, after which the bath was gradually warmed to $37^{\circ} \mathrm{C}$. Once the temperature was stable, a 30 -min equilibration period was allowed before taking any measurements. Images of Af-Arts were displayed at magnifications up to $1,980 \times$ and recorded with a video system consisting of a camera (CS520MD; Olympus), monitor (PVM1445MD; Sony, Tokyo) and video recorder (HR-S101; Victor, Tokyo). Using this system, a change in luminal diameter as small as $0.5 \mu \mathrm{m}$ is detectable. The diameter at the most responsive point was measured with a video micrometer (VM-30; Olympus).

\section{Experimental Protocols}

Protocol 1 : Effect of Ang II on the Luminal Diameter of AfArt

After the 30-min equilibration period, increasing doses of Ang II $\left(10^{-11}\right.$ to $10^{-8} \mathrm{M}$, Sigma) were added to both the bath and lumen. Luminal diameter was measured immediately before adding Ang II and observed for at least $10 \mathrm{~min}$ at each dose.

\section{Protocol 2: Effect of Miconazole (Mico) on Ang II Action}

We examined the possible contribution of endogenous EETs to Ang II-induced constriction by blocking its synthesis with an epoxygenase inhibitor Mico (Sigma) at $10^{-6} \mathrm{M}$, which selectively inhibits the formation of EETs by about $90 \%$ in rat preglomerular microvessels incubated with AA (13). After the equilibration period, Af-Arts were treated for $30 \mathrm{~min}$ with Mico added to both the bath and lumen. Effects of Ang II were then examined as in protocol 1 .

Protocol 3: Effect of 17-Octadecynoic Acid (17-ODYA) on Ang II Action

We next examined the possible contribution of endogenous CYP450 hydroxylase metabolites to Ang II action. Since the specific inhibitor for hydroxylase, which metabolizes AA to 19- and 20-HETE, is commercially unavailable, we used 17 ODYA $\left(10^{-6} \mathrm{M}\right.$; Sigma), an inhibitor for both epoxygenase and hydroxylase. In rat preglomerular microvessels incubated with AA, this concentration of 17-ODYA is reported to inhibit the formation of EETs and 20-HETE by about $85 \%$ and $90 \%$, respectively (13). After the equilibration period, Af-Arts were treated for $30 \mathrm{~min}$ with 17-ODYA added to both the bath and lumen. Effects of Ang II were then examined as in protocol 1 .

Protocol 4: Effect of Angiotensin II Type 2 Receptor (AT2R) Blockade

We have previously suggested the possibility that activation of $\mathrm{AT}_{2} \mathrm{R}$ is coupled to EETs synthesis in Af-Arts (14). In order to examine the possible involvement of $\mathrm{AT}_{2} \mathrm{R}$ in the contribution of CYP450-AA to Ang II action, protocols 1-3 were repeated in the presence of PD 123319 (PD; Research Biochemicals International, Natick, USA), a specific $\mathrm{AT}_{2} \mathrm{R}$ antagonist. After the equilibration period, $\mathrm{PD}$ at $10^{-7} \mathrm{M}$ was added to both the bath and lumen. Thirty minutes later, protocols 1-3 were performed. This dose of PD has been demonstrated to be specific for $\mathrm{AT}_{2} \mathrm{R}$ (15) and completely inhibit the $\mathrm{AT}_{2} \mathrm{R}$-mediated dilation in rabbit Af-Arts (14).

\section{Protocol 5: Effect of Mico or 17-ODYA on Norepinephrine} (NE)

We found that pretreatment with Mico or 17-ODYA augmented or attenuated Ang II action, respectively, suggesting that EETs modulate but CYP450 hydroxylase metabolites mediate Ang II action (see Results). To study whether such 


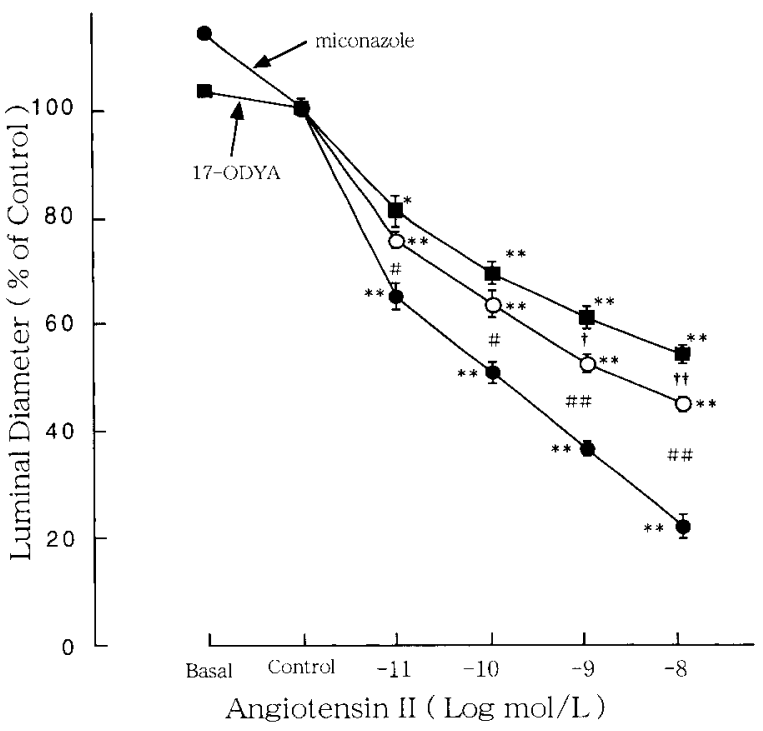

Fig. 1. Effects of miconazole or 17-ODYA on basal diameter and Ang II-induced constriction in Af-Arts. Luminal diameter is expressed as a percent of the pre-Ang II value (Control). Ang II constricted non-treated Af-Arts in a dosedependent manner $(\mathrm{O}, \mathrm{n}=9)$ ). Pretreatment with miconazole, a CYP450 epoxygenase inhibitor, at $10^{-6}$ moln significantly decreased basal diameter and augmented Ang II action (-) $\mathrm{n}=6$ ). On the other hand, pretreatment with 17-ODYA, which inhibits both CYP450 epoxygenase and hydroxylase, at $10^{-6} \mathrm{~mol} / \mathrm{l}$ had no effect on basal diameter but attenuated Ang II action ( $\mathbf{\square}, \mathrm{n}=6$ ). 17-ODYA, 17-octadecynoic acid; Ang II, angiotensin II; Af-Arts, afferent arterioles; CYP450, cytochrome P450. ${ }^{*} \mathrm{p}<0.01,{ }^{* *} \mathrm{p}<0.0001$ vs. control value. $\# \mathrm{p}<0.01, \# \mathrm{p}<0.0001$, miconazole-treated vs. non-treated group. ${ }^{\mathrm{t}} \mathrm{p}<0.01,{ }^{\dagger \dagger} \mathrm{p}<0.005,17-$ ODYA-treated vs. non-treated group.

effects are specific to Ang II or common to other vasoconstrictors, we examined the effect of Mico and 17-ODYA on NE action. Protocols identical to 1-3 were performed except that NE ( $10^{-8}$ to $10^{-5} \mathrm{M}$; Sigma) was used instead of Ang II.

\section{Data Analysis}

Values were expressed as the means $\pm S E M$, and all statistical analyses were carried out using \% changes. Student's paired $t$-test was used to examine whether the diameter differed from the control value or pretreated value within each group. ANCOVA was used to examine whether dose-response curves differed between groups, and a two-sample $t$ test was used to examine whether the change in diameter at a given concentration differed between groups. A value of $p<$ $0.0125(0.05 / 4$; Bonferroni's adjustment for four doses) was considered to indicate statistical significance using Bonferroni's multiple comparisons adjustment.

\section{Results}

Protocol I : Effect of Ang II on the luminal diameter of AfArt

The basal luminal diameter of Af-Arts was $15.9 \pm 0.4 \mu \mathrm{m}$ $(n=9)$. As shown in Fig. I, Ang II decreased the diameter in a dose-dependent manner, with a significant $(p<0.0001)$ constriction occurring from $10^{-11} \mathrm{M}$. Ang II at $10^{-11}, 10^{-10}$, $10^{-9}$ and $10^{-8} \mathrm{M}$ decreased the diameter to $71 \pm 2 \%, 56 \pm$ $3 \%, 43 \pm 2 \%$ and $34 \pm 2 \%$ of the control diameter, respectively.

\section{Protocol 2: Effect of Mico on Ang II Action}

Pretreatment with Mico decreased the luminal diameter from $15.4 \pm 0.5$ to $13.2 \pm 0.6 \mu \mathrm{m}(n=6, p<0.0001)$ and significantly $(p<0.01)$ augmented Ang II action. In Af-Arts pretreated with Mico, Ang II at $10^{-11}, 10^{-10}, 10^{-9}$ and $10^{8} \mathrm{M}$ decreased the diameter to $58 \pm 3 \%, 41 \pm 3 \%, 25 \pm 2 \%$ and $7 \pm 3 \%$ of control the diameter, respectively (Fig. 1).

Protocol 3: Effect of 17-ODYA on Ang II Action

Pretreatment with 17-ODYA did not affect the basal luminal diameter; the diameters before and after the treatment were $16.0 \pm 0.6$ and $15.4 \pm 0.7 \mu \mathrm{m}(n=6)$, respectively. In AfArts pretreated with 17-ODYA, Ang II at $10^{-11}, 10^{-10}, 10^{-9}$ and $10^{-8} \mathrm{M}$ decreased the diameter to $77 \pm 3 \%, 63 \pm 3 \%$, $53 \pm 2 \%$ and $46 \pm 2 \%$ of the control diameter, respectively (Fig. 1). The decrease in diameter induced by Ang II was significantly $(p<0.01)$ attenuated by 17-ODYA at $10^{9}$ and $10^{-8} \mathrm{M}$.

\section{Protocol 4: Effect of $A T_{2} R$ Blockade}

Pretreatment with PD did not affect the basal luminal diameter; the diameters before and after the treatment were $16.5 \pm$ 0.7 and $16.2 \pm 0.7 \mu \mathrm{m}(n=6)$, respectively. In Af-Arts pretreated with PD, Ang II at $10^{-11}, 10^{-10}, 10^{-9}$ and $10^{-8} \mathrm{M}$ decreased the diameter to $70 \pm 4 \%, 50 \pm 5 \%, 32 \pm 4 \%$ and $19 \pm$ $2 \%$ of the control diameter, respectively (Fig. 2). The decrease in diameter induced by Ang II was significantly $(p<$ 0.005 ) augmented by PD at $10^{-9}$ and $10^{-8} \mathrm{M}$. Pretreatment with both PD and Mico decreased the luminal diameter from $16.0 \pm 0.3$ to $14.2 \pm 0.4 \mu \mathrm{m}(n=6, p<0.0001)$, which was not different from that induced by Mico alone. In Af-Arts pretreated with both PD and Mico, Ang II at $10^{-11}, 10^{-10}$, $10^{-9}$ and $10^{-8} \mathrm{M}$ decreased the diameter to $65 \pm 4 \%, 48 \pm$ $3 \%, 30 \pm 3 \%$ and $11 \pm 4 \%$ of the control diameter, respectively (Fig. 2). The decrease in diameter induced by Ang II was not different from those observed in Af-Arts pretreated with PD alone, demonstrating that EETs synthesis inhibition with Mico does not augment Ang II action in Af-Arts with $\mathrm{AT}_{2} \mathrm{R}$ blockade. Pretreatment with both $\mathrm{PD}$ and 17-ODYA did not affect the basal luminal diameter; the diameters before and after the treatment were $15.0 \pm 0.7$ and $14.7 \pm 0.6$ $\mu \mathrm{m}(n=6)$, respectively. In Af-Arts pretreated with both PD 


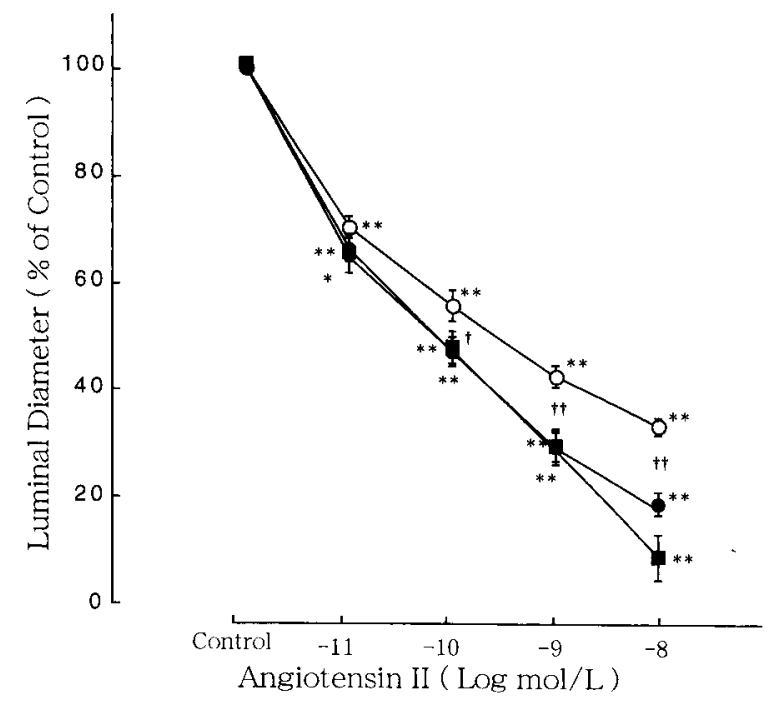

Fig. 2. Effects of miconazole on Ang II-induced constriction in PD-treated Af-Arts. PD, an Ang II type 2 receptor antagonist, at $10^{-7} \mathrm{~mol} / \mathrm{l}$ significantly augmented Ang II action ( $\mathrm{n}=6)$ compared to that observed in non-treated Af-Arts $(O$, $\mathrm{n}=6)$. In the Af-Arts pretreated with $P D$, miconazole did not further augment Ang II action $(\mathbf{E}, \mathrm{n}=6)$. Ang II, angiotensin II; PD, PD 123319; Af-Arts, afferent arterioles. ${ }^{*} \mathrm{p}<0.01$, ${ }^{* *} \mathrm{p}<0.0001$ vs. control value. ${ }^{\dagger} \mathrm{p}<0.005,{ }^{{ }^{\dagger}} \mathrm{p}<0.0001$, miconazole-treated vs. PD-treated group.

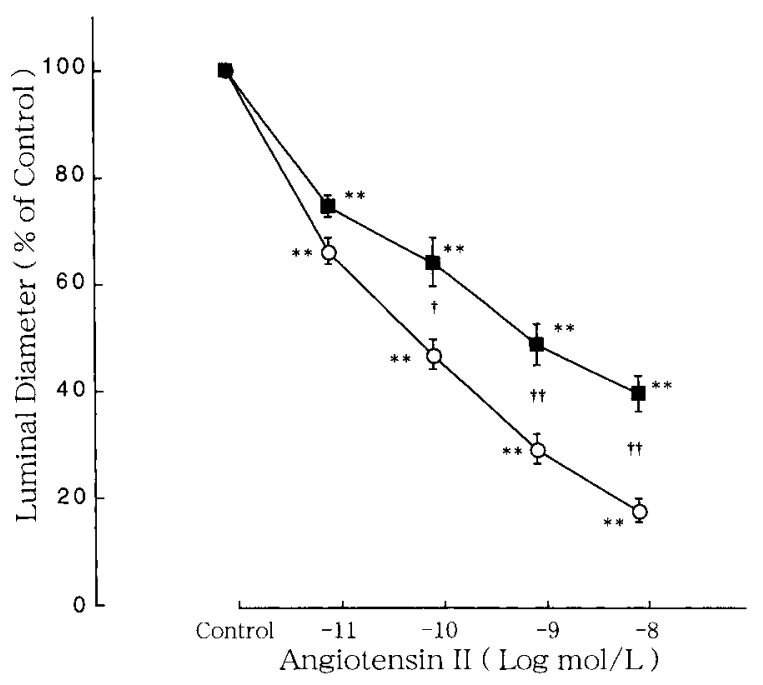

Fig. 3. Effects of 17-ODYA on Ang II-induced constriction in PD-treated Af-Arts. Pretreatment with 17-ODYA (, $\mathrm{n}=$ 6) significantly attenuated vasoconstrictor action of Ang II in Af-Arts with Ang II type 2 receptor blockade $(\mathrm{O}, \mathrm{n}=6) .17$ ODYA, 17-octadecynoic acid; Ang II, angiotensin II; PD, $P D$ 123319; Af-Arts, afferent arterioles. ${ }^{* *} \mathrm{p}<0.0001$ vs. control value. ${ }^{\dagger} \mathrm{p}<0.005,{ }^{\dagger+} \mathrm{p}<0.0005,17-O D Y A$ plus $P D-$ treated vs. $P D$-treated group.

and 17-ODYA, Ang II at $10^{-11}, 10^{-10}, 10^{-9}$ and $10^{-8} \mathrm{M}$ decreased the diameter to $75 \pm 2 \%, 65 \pm 5 \%, 50 \pm 4 \%$ and $41 \pm$ $3 \%$ of the control diameter, respectively (Fig. 3). The de-

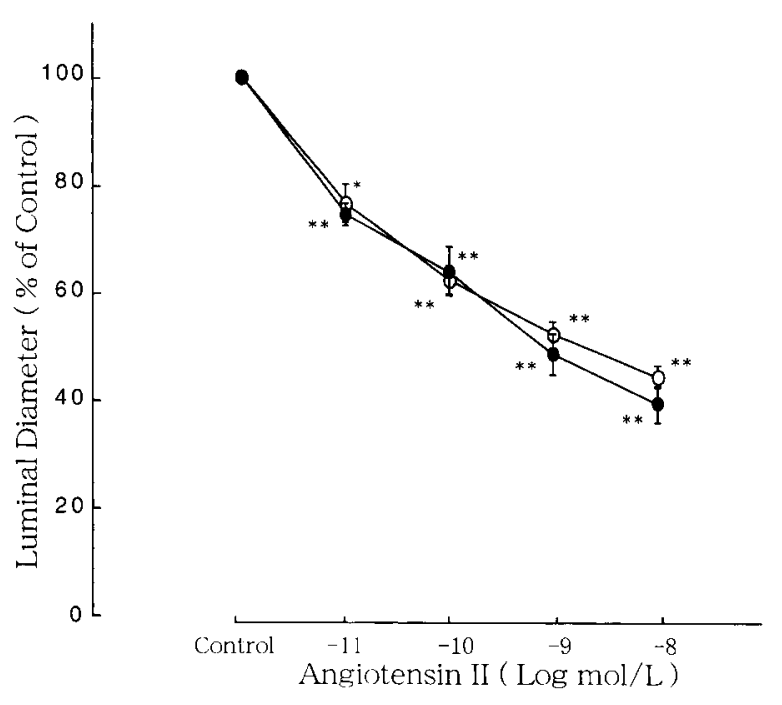

Fig. 4. Effects of Ang II type 2 receptor blockade with $P D$ in 17-ODYA-treated Af-Arts. $P D$ at $10^{-7} \mathrm{~mol} / \mathrm{l}(\mathbf{O}, \mathrm{n}=6)$ had no effect on Ang II action in Af-Arts pretreated with 17 ODYA (O, $\mathrm{n}=6)$. Ang II, angiotensin II; PD, PD 123319; 17-ODYA, 17-octadecynoic acid; Af-Arts, afferent arterioles. ${ }^{*} \mathrm{p}<0.01,{ }^{* *} \mathrm{p}<0.0001$ vs. control value.

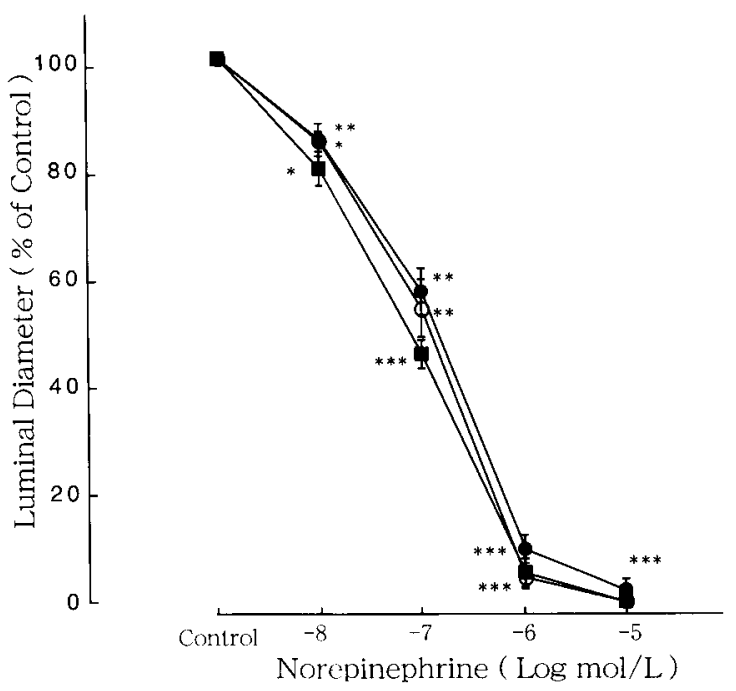

Fig. 5. Effects of miconasole or 17-ODYA on NE-induced constriction in Af-Arts. NE constricted non-treated Af-Arts in a dose-dependent manner $(\bigcirc, \mathrm{n}=6)$. Pretreatment with either miconazole $(\mathbf{O}, \mathrm{n}=6)$ or 17-ODYA $(\mathbf{\square}, \mathrm{n}=6)$ at $10^{-6}$ mol/l had no effect on NE action. 17-ODYA, 17-octadecynoic acid; NE, norepinephrine; Af-Arts, afferent arterioles. ${ }^{*} \mathrm{p}<$ $0.01,{ }^{* *} \mathrm{p}<0.001,{ }^{* * *} \mathrm{p}<0.0001$ vs. control value.

crease in diameter induced by Ang II was significantly ( $p<$ 0.01 ) attenuated by 17 -ODYA at $10^{-10}, 10^{-9}$ and $10^{-8} \mathrm{M}$ in the presence of PD. Comparing the dose-response curves ob- 
tained in Af-Art treated with 17-ODYA alone and in those treated with 17-ODYA plus PD demonstrates that PD had no further effect in the presence of 17-ODYA (Fig. 4).

\section{Protocol 5: Effect of Mico or 17-ODYA on NE Action}

The basal luminal diameter of Af-Art was $15.9 \pm 0.4 \mu \mathrm{m}$ $(n=6)$. NE decreased the diameter in a dose-dependent manner (Fig. 5). Significant constriction was observed from $10^{-8}$ M $(15 \pm 3 \%, p<0.01)$. In marked contrast to Ang II-induced constriction, neither Mico nor 17-ODYA affected NE-induced constriction in Af-Arts (Fig. 5).

\section{Discussion}

Recent studies indicate that CYP450-AA may play physiological roles in the regulation of renal hemodynamics $(8,16)$. Inhibition of CYP450 hydroxylase impairs the autoregulation of renal blood flow (13), whereas EETs dilate renal microvessels (17). Imig et al. (18) also demonstrated that CYP450 epoxygenase and hydroxylase pathways contribute to the autoregulatory response of the Af-Art, a vascular segment crucial to the control of RVR. In the present study, we examined the contribution of these CYP450-AA to the action of Ang II on isolated microperfused rabbit Af-Arts, and found that 1) the vasoconstrictor action of Ang II, but not that of NE, was significantly augmented by Mico and attenuated by 17-ODYA, and 2) the effect of Mico, but not that of 17-ODYA, was abolished by $\mathrm{AT}_{2} \mathrm{R}$ blockade with PD. Our results demonstrate that in the Af-Art, endogenous CYP450$\mathrm{AA}$ are involved in the vasoconstrictor action of Ang II, but not that of NE. Furthermore, it appears that CYP 450 epoxygenase pathway may attenuate Ang II action via $\mathrm{AT}_{2}$ receptors.

Although our results clearly demonstrate the contribution of CYP450 hydroxylase metabolites to the vasoconstrictor action of Ang II, it remains to be determined which metabolites are involved. Among several HETEs, 19- and 20-HETE are known to be the primary CYP450 hydroxylase products in the kidney (19). It has been demonstrated that Ang II increases the release of 20-HETE in the Af-Art (7) and that 20HETE constricts rat and rabbit Af-Arts $(9,20)$, whereas 19HETE has been reported not to cause vasoconstriction in the kidney $(21,22)$. Taken together, these results strongly suggest that in the Af-Art, Ang II stimulates the production of 20-HETE, which in turn mediates the vasoconstrictor action of Ang II on this vascular segment. In accordance with this notion, Alonso-Galicia et al. (23) reported that Ang II increases renal production of 20-HETE and that increased 20HETE contributes to the renal vasoconstrictor and pressor actions of Ang II.

We here found that inhibition of EETs synthesis by Mico augmented Ang II-induced constriction, while inhibition of both EETs and hydroxylase metabolites by 17-ODYA attenuated Ang II-induced constriction. These results suggest that endogenous EETs or hydroxylase metabolites attenuate or potentiate Ang II action in Af-Arts, respectively, and that the influences of hydroxylase metabolites overcome those of EETs. In contrast, Imig and Deichmann (24) have reported that pretreatment with 17-ODYA as well as Mico augments Ang II-induced constriction in rat juxtamedullary Af-Arts. Oyekan et al. (25) also reported that specific inhibition of renal 20-HETE production with 12,12-dibromododec-11-enoic acid (which is not commercially available) does not affect Ang II-induced constriction in the rat isolated perfused kidney. These studies suggest that the influences of 20-HETE on Ang II action, if any, are less pronounced than those of EETs. Although the reason for this discrepancy is unclear, there are several possibilities other than species differences (rabbit $v s$. rat). First, it may be related to the difference in the preparation used (particularly the presence or absence of proximal tubules, the major site of EETs production in the kidney (26)). We have previously demonstrated that Ang II stimulates the production of EETs to a much greater degree than does 20-HETE in the proximal tubules (26). Thus, it is possible that their preparation included much greater amounts of EETs (which would attenuate the vasoconstrictor action of Ang II) than did ours. Second, since we used isolated Af-Arts, we could not observe the effect of TGF that contributes to the control of Af-Art resistance in Imig's preparation (juxtamedullary nephron preparation) or Oyekan's preparation (isolated perfused kidney) in which tubular and vascular relations are preserved. 20-HETE is known to inhibit $\mathrm{Na}^{+}-\mathrm{K}^{+}-2 \mathrm{Cl}$ cotransport at the thick ascending limb of Henle and to affect the Af-Art resistance through TGF (8). Thus, in their studies, inhibition of 20-HETE synthesis may have indirectly affected the Af-Art resistance partly through the alteration in TGF, making it difficult to compare the vascular reactivity of Af-Art between our preparation and theirs.

In the present study, blockade of the $\mathrm{AT}_{2} \mathrm{R}$ with $\mathrm{PD}$ abolished the Mico-induced augmentation of Ang II action. This is consistent with our previous study (14), which suggested that activation of $\mathrm{AT}_{2} \mathrm{R}$ is coupled to EETs production in AfArts. In contrast, 17-ODYA (which inhibits both EETs and CYP450 hydroxylase metabolites synthesis) attenuated Ang II action in Af-Arts even in the presence of PD, suggesting that $\mathrm{AT}_{2} \mathrm{R}$ is not involved in the 17-ODYA-induced attenuation of Ang II action. Considering that blockade of $\mathrm{AT}_{2} \mathrm{R}$ with PD had the same effects on Ang II action as did EETs synthesis inhibition, this result reveals that PD does not influence the effect of CYP450 hydroxylase metabolites on Ang II action in Af-Arts, and suggests that CYP450 hydroxylase metabolites potentiate Ang II action through mechanisms other than AT2R. Since Ang II exerts its vascular actions solely through its type 1 receptor $\left(A T_{1} R\right)$ or $A T_{2} R$ in Af-Arts of our preparation (14), it may be that AT $R$ is involved in CYP450 hydroxyalase metabolites-mediated potentiation of Ang II action in this vascular segment. Schema illustrating the possible receptor subtype of Ang II that links to the CYP450-AA production in Af-Arts are shown in Fig. 6.

In addition to its effects on Ang II action, we also found 


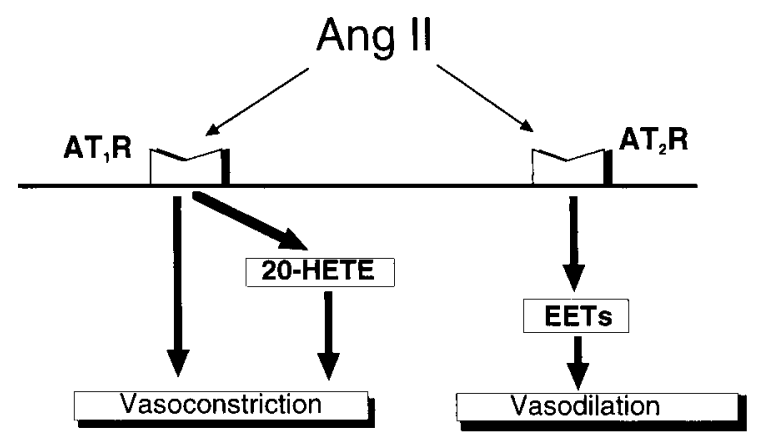

Fig. 6. Schematic illustration of the possible receptor subtype of Ang II that links to the CYP450-AA production in Af-Arts. In Af-Arts, Ang II may stimulate 20-HETE production through $A T_{1} R$, while it may stimulate EETs production through $A T_{2} R$. Ang II, angiotensin II; CYP450, cytochrome P450; 20-HETE, 20-hydroxyeicosatetraenoic acid; ATIR, angiotensin II type I receptor; EETs, epoxyeicosatrienoic acids; $A T_{2} R$, angiotensin II type 2 receptor; Af-Arts, afferent arterioles.

that tonic release of CYP450-AA plays a role in the control of the basal resting tone of Af-Arts. EETs synthesis inhibition with Mico significantly decreased the basal diameter of Af-Arts, whereas both EETs and CYP450 hydroxylase metabolites synthesis inhibition with 17-ODYA had no effect, demonstrating that EETs decrease but CYP450 hydroxylase metabolites increase the basal Af-Art tone. The effect of EETs synthesis inhibition on Af-Arts is found to be similar to the effect of NO synthesis inhibition we reported previously (27); both decrease basal diameter and augment Ang II but not NE action. These results indicate that EETs and NO share similar characteristics in the control of Af-Arts tone and that there may be an interaction between EETs and NO. The latter possibility is supported by the findings of AlonsoGalicia et al. (28) and Ito et al. (29). These authors found that an increase in NO level inhibits the activity of CYP450 epoxygenase and hence the production of EETs in preglomerular microvessels and glomeruli. These findings suggest that, under pathophysiological conditions with decreased NO synthesis, the release of EETs may be increased to compensate for the impaired vasodilator action of NO in the kidney.

In summary, we have shown that in isolated microperfused rabbit Af-Arts, EETs synthesis inhibition with Mico decreases the luminal diameter, whereas EETs and CYP450 hydroxylase metabolites synthesis inhibition with 17-ODYA has no effect on it. We also found that the constrictor action of Ang II on Af-Arts is significantly augmented by Mico and attenuated by 17-ODYA, and that the effect of Mico but not 17-ODYA on Ang II action was abolished by $\mathrm{AT}_{2} \mathrm{R}$ blockade. While the present results were obtained by pharmacological interventions and the study has some limitations, our findings may provide evidence that endogenous CYP450AA are involved in the control of the basal vascular tone of Af-Arts, as well as the vasoconstrictor action of Ang II on
Af-Arts (in particular, the CYP450 epoxygenase pathway attenuates Ang II action via $\mathrm{AT}_{2} \mathrm{R}$ ).

\section{Acknowledgements}

The authors thank Miss Hiromi Takahashi and Miss Yoshiko Takita for their secretarial assistance and Mr. Katsuyuki Tanaka for his experimental assistance.

\section{References}

1. Ichikawa I, Harris RC: Angiotensin actions in the kidney: renewed insight into the old hormone. Kidney Int 1991; 40: 583-596.

2. Arendshorst WJ, Chatziantoniou C, Daniels FH: Role of angiotensin in the renal vasoconstriction observed during the development of genetic hypertension. Kidney Int 1990; 30 (Suppl): S92-S96.

3. Navar LG, Inscho EW, Majid SA, Imig JD, HarrisonBernard LM, Mitchell KD: Paracrine regulation of the renal microcirculation. Physiol Rev 1996; 76: 425-536.

4. Ito S, Carretero OA, Abe K: Role of nitric oxide in the control of glomerular microcirculation. Clin Exp Pharmacol Physiol 1997; 24: 578-581.

5. Deng X, Welch WJ, Wilcox CS: Role of nitric oxide in short-term and prolonged effects of angiotensin II on renal hemodynamics. Hypertension 1996; 27: 1173-1179.

6. Harder DR, Lange AR, Gebremedhin D, Birks EK, Roman RJ: Cytochrome P450 metabolites of arachidonic acid as intracellular signaling molecules in vascular tissue. $J$ Vasc Res 1997; 34: 237-243.

7. Croft KD, McGiff JC, Sanchez-Mendoza A, Carroll MA: Angiotensin II releases 20-HETE from rat renal microvessels. Am J Physiol 2000; 279: F544-F551.

8. Zou AP, Imig JD, Ortiz de Montellano PR, Sui Z, Falck JR, Roman RJ: Effect of P-450 omega-hydroxylase metabolites of arachidonic acid on tubuloglomerular feedback. Am J Physiol 1994; 266: F934-F941.

9. Arima S, Omata K, Ito S, Tsunoda K, Abe K: 20-HETE requires increased vascular tone to constrict rabbit afferent arterioles. Hypertension 1996; 27: 781-785.

10. Ito $\mathrm{S}$ and Carretero $\mathrm{OA}$ : An in vitro approach to the study of macula densa-mediated glomerular hemodynamics. Kidney Int 1990; 38: 1206-1210.

11. Ito S, Johnson CS, Carretero OA: Modulation of angiotensin II-induced vasoconstriction by endothelium-derived relaxing factor in the isolated microperfused rabbit afferent arteriole. J Clin Invest 1991; 87: 1656-1663.

12. Arima $\mathrm{S}$, Ito $\mathrm{S}$, Omata $\mathrm{K}$, Takeuchi $\mathrm{K}$, Abe K: High glucose augments angiotensin II action by inhibiting NO synthesis in in vitro microperfused rabbit afferent arterioles. Kidney Int 1995; 48: 683-689.

13. Zou AP, Imig JD, Kaldunski M, Ortiz de Montellano PR, Sui Z, Roman RJ: Inhibition of renal vascular 20-HETE production impairs autoregulation of renal blood flow. Am J Physiol 1994; 266: F275-F282.

14. Arima S, Endo $\mathrm{Y}$, Yaoita $\mathrm{H}$, et al: Possible role of P-450 metabolite of arachidonic acid in vasodilator mechanism of angiotensin II type 2 receptor in the isolated microperfused 
rabbit afferent arteriole. J Clin Invest 1997; 100: 2816-2823.

15. Brechler V, Jones PW, Levens NR, de Gasparo M, Bottari SP: Agonistic and antagonistic properties of angiotensin analogs at the AT2 receptor in PC12W cells. Regul Pept 1993; 44: 207-213.

16. Kauser K, Clark JE, Masters BS, et al: Inhibitors of cytochrome P-450 attenuate the myogenic response of dog renal arcuate arteries. Circ Res 1991; 68: 1154-1163.

17. Imig JD, Navar LG, Roman RJ, Reddy KK, Falck JR: Actions of epoxygenase metabolites on the preglomerular vasculature. J Am Soc Nephrol 1996; 7: 2364-2370.

18. Imig JD, Falck JR, Inscho EW: Contribution of cytochrome P450 epoxygenase and hydroxylase pathways to afferent arteriolar autoregulatory responsiveness. $\mathrm{Br} J$ Pharmacol 1999; 127: 1399-1405.

19. Carroll MA, Balazy M, Margiotta P, Huang DD, Falck JR, McGiff JC: Cytochrome P-450-dependent HETEs: profile of biological activity and stimulation by vasoactive peptides. Am J Physiol 1996; 271: R863-R869.

20. Imig JD, Zou AP, Stec DE, Harder DR, Falck JR, Roman RJ: Formation and actions of 20-hydroxyeicosatetraenoic acid in rat renal arterioles. Am J Physiol 1996; 270: R217-R227.

21. Carroll MA, Garcia MP, Falck JR, McGiff JC: Cyclooxygenase dependency of the renovascular actions of cytochrome P450-derived arachidonate metabolites. J Pharmacol Exp Ther 1992; 260: 104-109.

22. Ma YH, Gebremedhin D, Schwartzman ML, et al: 20-Hy- droxyeicosatetraenoic acid is an endogenous vasoconstrictor of canine renal arcuate arteries. Circ Res 1993; 72: 126-136.

23. Alonso-Galicia M, Falck JR, Reddy KM, Roman RJ: 20HETE agonists and antagonists in the renal circulation. $\mathrm{Am}$ J Physiol 1999; 277: F790-F796.

24. Imig JD, Deichmann PC: Afferent arteriolar responses to ANG II involve activation of PLA2 and modulation by lipoxygenase and P-450 pathways. Am J Physiol 1997; 273: F274-F282.

25. Oyekan A, Balazy M, McGiff JC: Renal oxygenases: differential contribution to vasoconstriction induced by ET-1 and ANG II. Am J Physiol 1997; 273: R293-R300.

26. Omata K, Abraham NG, Schwartzman ML: Renal cytochrome P-450-arachidonic acid metabolism: localization and hormonal regulation in SHR. Am J Physiol 1992; 262: F591-F599.

27. Ito S, Arima S, Ren YL, Juncos LA, Carretero OA: Endotherium-derived relaxing factor/nitric oxide modulates angiotensin Il action in the isolated microperfused rabbit afferent but efferent arteriole. J Clin Invest 1993; 91: 3012-3019.

28. Alonso-Galicia M, Sun CW, Falck JR, Harder DR, Roman RJ: Contribution of 20-HETE to the vasodilator actions of nitric oxide in renal arteries. Am J Physiol 1998; 275: F370-F378.

29. Ito O, Roman RJ: Regulation of P-450 4A activity in the glomerulus of the rat. Am J Physiol 1999; 276: R1749-R1757. 